\title{
Tuning Transparent Supercapacitor Performance by Controlling the Morphology of its $\mathrm{ZnO}$ Electrodes
}

\author{
M.A. BorysieWicz ${ }^{a, *}$, M. WzOreK ${ }^{a}$, M. EkIElski $^{a}$, J. KACZMARski $^{a}$ \\ AND T. WOJCIECHOWSKI ${ }^{b}$ \\ ${ }^{a}$ Institute of Electron Technology, al. Lotników 32/46, 02-668 Warsaw, Poland \\ ${ }^{b}$ Institute of Physics, Polish Academy of Sciences and International Research Centre MagTop, \\ Aleja Lotnikow 32/46, PL-02668 Warsaw, Poland \\ (Received December 29, 2016)
}

\begin{abstract}
Transparent supercapacitors were fabricated using nanostructures $\mathrm{ZnO}$ electrodes deposited using reactive magnetron sputtering. By fine tuning the deposition process parameters the electrodes with different morphologies were obtained, from hierarchical through sponge-like to nanocolumnar. The device performance related to the electrode morphology was assessed. It was found that the nanocolumnar electrodes provided best performance both in terms of effective device capacitance $\left(18.3 \mu \mathrm{F} / \mathrm{cm}^{2}\right)$ and transparency $(\approx 100 \%$ in the visible range). The worst performance, with $80 \%$ lower effective capacitance, was obtained in the devices with the sponge-like morphology.
\end{abstract}

DOI: $10.12693 /$ APhysPolA.131.1550

PACS/topics: 82.47.Uv, 68.35.bg, 68.55.ag, 81.15.Cd

\section{Introduction}

Transparent electronics is a new and rapidly developing area where the electronic devices are needed not only to perform their normal functions (displays, controllers, I/O devices, energy storage etc.) but also need to be transparent for aesthetic reasons. The possible applications in personal, wearable or architectural devices makes such devices appealing for both the end user as well as the researcher. With the most focus on the development of transparent displays, work on transparent energy storage devices has been limited. In the case of supercapacitors, the most used approach for transparent electrodes is to apply very thin carbon nanostructured films, combining porosity and transparency. Such devices offer effective capacitances of the order of $1 \mathrm{mF} / \mathrm{cm}^{2}$ with a transparency of around $50 \%$ at $550 \mathrm{~nm}$ [1-3].

Another approach is to apply transparent oxide semiconductor nanostructures as the electrodes in order to benefit from their intrinsic transparency $[4,5]$. Following this way, thicker electrodes can be realized and if their porosities and morphologies are tailored, the performance could in the future overcome that of the carbon-based devices.

We study transparent supercapacitor electrodes fabricated of wide band gap zinc oxide $(\mathrm{ZnO})$ nanostructured thin films deposited by reactive magnetron sputtering with postdeposition annealing [6]. We have previously shown that by appropriate fine control of the process gas flow ratios and values we can control the morphology of such nanocrystalline $\mathrm{ZnO}$ in a significant range [7]. In this report, we utilize this possibility to fabricate

\footnotetext{
*corresponding author; e-mail: mbory@ite.waw.pl
}

in a controlled manner $\mathrm{ZnO}$ electrodes with different morphologies and to assess their performance directly in transparent supercapacitor devices.

\section{Experimental}

Nanostructured $\mathrm{ZnO}$ thin films were prepared by magnetron sputter deposition of a $\mathrm{Zn}$ target in a reactive $\mathrm{Ar} / \mathrm{O}_{2}$ gas mixture. The $75 \mathrm{~mm}$ target was under $80 \mathrm{~W}$ DC power and the substrates were not intentionally heated. The argon and oxygen flow values were selected so that various nanostructure morphologies could be obtained [7]. The Ar: $\mathrm{O}_{2}$ flows in sccm were respectively: 3:0.3, 3:0.6, 10:1, 10:2, 20:2, and 20:4. The samples will be identified by these flow values. All deposited films except the "20:4" were in fact zinc nanostructures with surface covered by a native oxide [7], therefore in order to obtain $\mathrm{ZnO}$ nanostructures they had to be oxidized in a furnace. A Mattson SHS 100 rapid thermal processing furnace was used, where the nanostructures were oxidized in pure oxygen for $5 \mathrm{~min}$ at $400^{\circ} \mathrm{C}$. Subsequently, all samples were doped with hydrogen to increase their conductivity, as $\mathrm{H}$ is regarded as a shallow donor in $\mathrm{ZnO}$ [8]. Hydrogen was introduced by annealing in an $\mathrm{Ar} / \mathrm{H}_{2}$ mixture for $30 \mathrm{~s}$ at $350^{\circ} \mathrm{C}$. Scanning electron microscopy (SEM) imaging on a Zeiss Neon 40 was performed to assess the morphology type of the $\mathrm{ZnO}$ nanostructures fabricated on silicon substrates. Supercapacitors were assembled with $700 \mathrm{~nm}$ thick electrodes deposited on fluorine-doped indium tin oxide-covered glass slides. A shadow mask was used for Zn deposition to determine the surface area as $1 \mathrm{~cm}^{2}$. Each supercapacitor was fabricated from two symmetrical electrodes, a $100 \mu \mathrm{m}$ thick gasket for sealing the electrolyte and a $\mathrm{LiCl}$ gel-based electrolyte [9]. The completed devices were tested by using a Gamry Reference 3000 potentiostat. We performed cyclic voltammetry, cyclic charge/discharge, and electrochemical 
impedance spectroscopy (EIS) measurements. Optical spectral transmission of the devices was measured using a Woolam M2000 ellipsometer.

\section{Results and discussion}

The cross-section SEM images of the samples studied in this experiment are shown in Fig. 1. It can be seen that for the lowest flow values the nanocrystals as well as the pores are relatively large. By increasing the oxygen flow and maintaining the set flow ratios, we obtain a reduction in both pore sizes and nanocrystal sizes, up to the sample "10:2". For the sample "20:2" an increase in pore sizes is apparent and the sample "20:4" has a nanocolumnar morphology and thus larger pores that are the spaces between the columns.

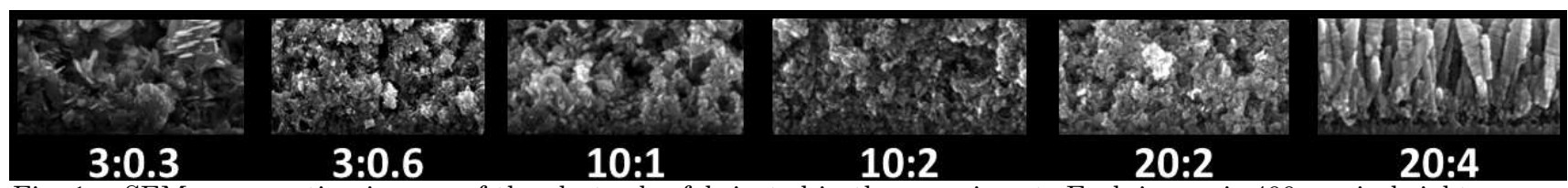

Fig. 1. SEM cross-section images of the electrodes fabricated in the experiment. Each image is $400 \mathrm{~nm}$ in height.
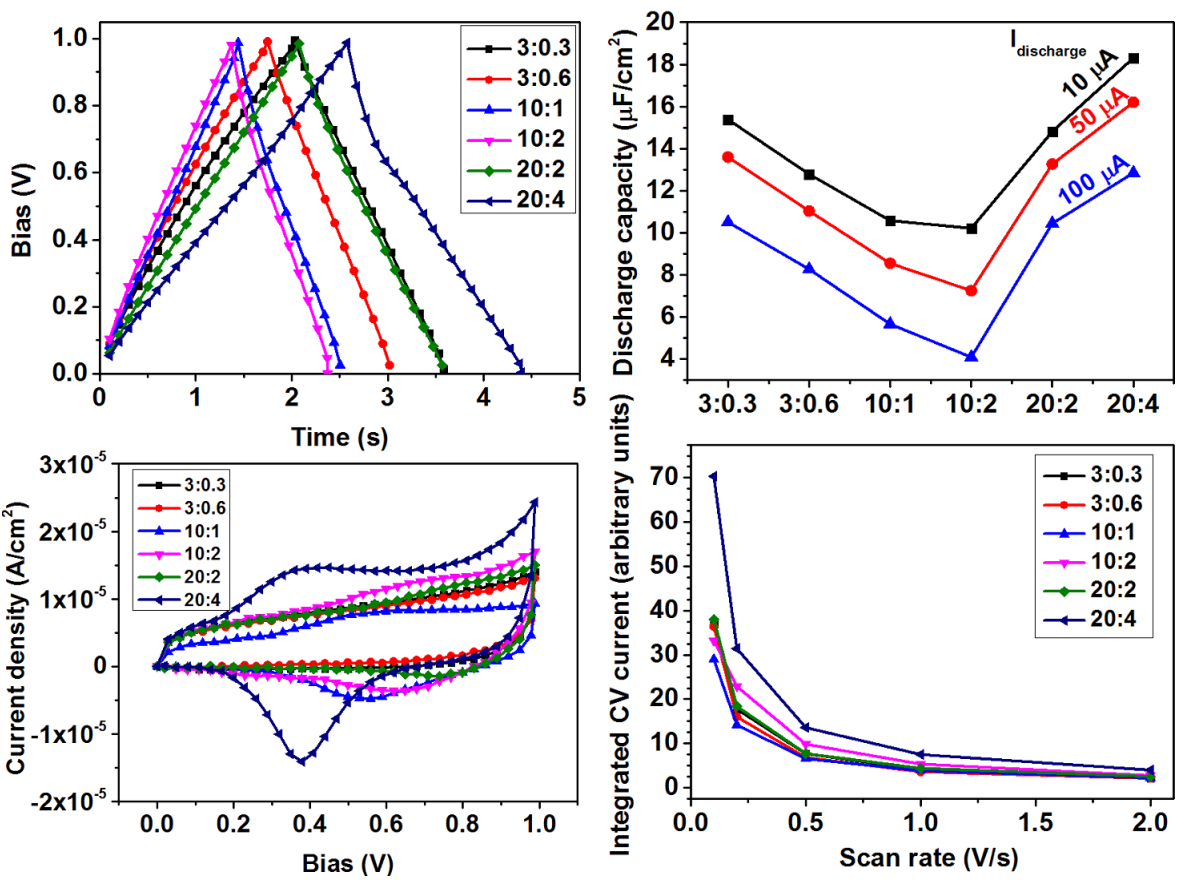

Fig. 2. Electrochemical characteristics of the supercapacitors: (a) charge/discharge curves at $10 \mu \mathrm{A}$ constant current, (b) effective discharge capacity as a function of the charge/discharge current, (c) cyclic voltammetry scans, (d) cyclic voltammetry integrated current as a function of the voltage scan rate.

The devices were tested first by charging and discharging at constant currents of 10,50 , and $100 \mu \mathrm{A}$. Figure $2 \mathrm{a}$ presents the charge and discharge curves as a function of time for $10 \mu \mathrm{A}$ current. The shape of all curves except the "20:4" is generally triangular, which can indicate an electrostatic charge storage mechanism, as expected from $\mathrm{ZnO}$. The effective capacitances $\left(C_{\text {eff }}\right)$ calculated from the discharge part of the charge-discharge curve are plotted in Fig. 2b. The $C_{\text {eff }}$ values are in the range 10.2 $18.3 \mu \mathrm{F} / \mathrm{cm}^{2}$, which means that by appropriate choice of the electrode morphology it is possible to gain an $80 \%$ increase in the capacitance of the devices. The $C_{\text {eff }}$ curve can be divided in two parts. First, starting at $C_{\text {eff }}=15.4 \mu \mathrm{F} / \mathrm{cm}^{2}$ for the sample " $3: 0.3$ " and decreasing in value until the sample "10:2". The second, for
"20:2" and "20:4" an increase in $C_{\text {eff }}$ up to $18.3 \mu \mathrm{F} / \mathrm{cm}^{2}$ for " $20: 4$ ". This can be related to the view that the optimal morphology for a supercapacitor should be a hierarchical one, where macropores branch out into mesopores and these in turn into nanopores [10]. If a morphology with small crystallites and very small pores is observed ("10:2") the solvated ion in the electrolyte is unable to effectively penetrate the thickness of the film and the usable surface area of the electrode is thus lowered. On the other hand, the morphologies with hierarchical structures, such as those observed for the other samples tend to have a higher capacitances, however the quantitative description of the optimal conditions is beyond the scope of this work. The sample "20:4" is a different case, where the macropores are aligned perpendicularly 
to the electrode surface, and at the same time in parallel with the external electric field due to voltage bias. In such a morphology, the electrolyte penetration is highly effective and the solvated ions can be transported to all of the available surface. It has to be noted here that the electrode sheet resistances were around 36-41 $\Omega / \square$ for all samples except "20:2", where the sheet resistance was the lowest at $29 \Omega / \square$. Such small sheet resistance changes could not induce the significant changes in the capacitance, and therefore, the latter can be attributed to the morphology of the electrodes.

The cyclic voltammetry scans shown in Fig. 2c for a bias voltage sweep rate of $1 \mathrm{~V} / \mathrm{s}$ have significant nonlinearities. For the samples "3:0.3" and "3:06" they resemble the ideal electrostatic capacitor box shape. For the other samples however, peaks in cathodic and anodic currents appear, the most visible for the sample "20:4". The presence of such peaks indicates electrochemical charge storage taking place in the devices. The cathodic and anodic peak separations are very small in each sample, however the intensities of the anodic peaks are larger than those of the cathodic ones, which points to an irreversible character of the reactions taking place. Integration of a cyclic voltammetry sweep and its normalization by bias scan rate and bias range yields a value proportional to the device capacitance. These values are presented in Fig. 2d as a function of the bias scan rate. The integral drops as the scan rate increases indicating that there are slow charge storage processes, most notable in the sample "20:4". These are most probably chemical reactions which could be related to the current peaks observed in cyclic voltammetry.

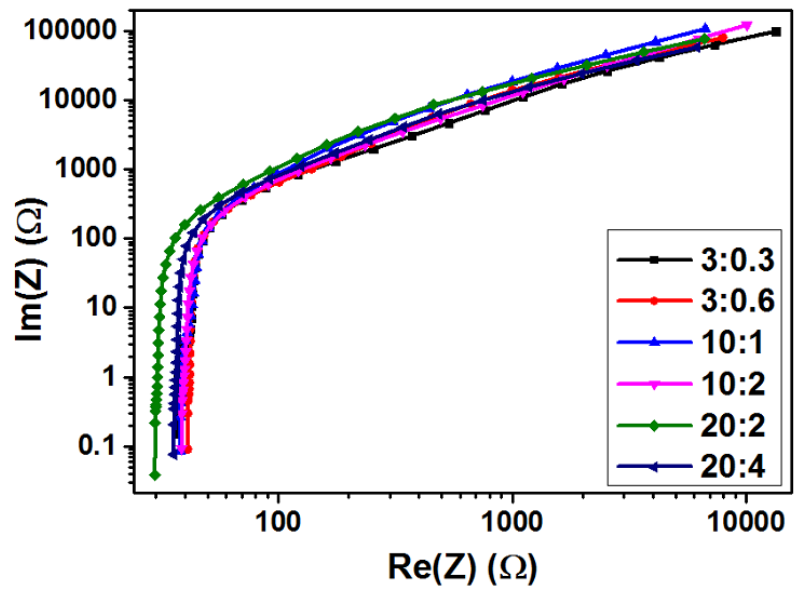

Fig. 3. Nyquist plot of the EIS spectra of the supercapacitors.

The EIS spectra are presented in Fig. 3. They can be described by a diffusion impedance in the low frequency/high impedance tail. The series resistances (ESR) were determined from these plots at high frequencies and were used to determine the power density of the supercapacitors, shown in Fig. 4.

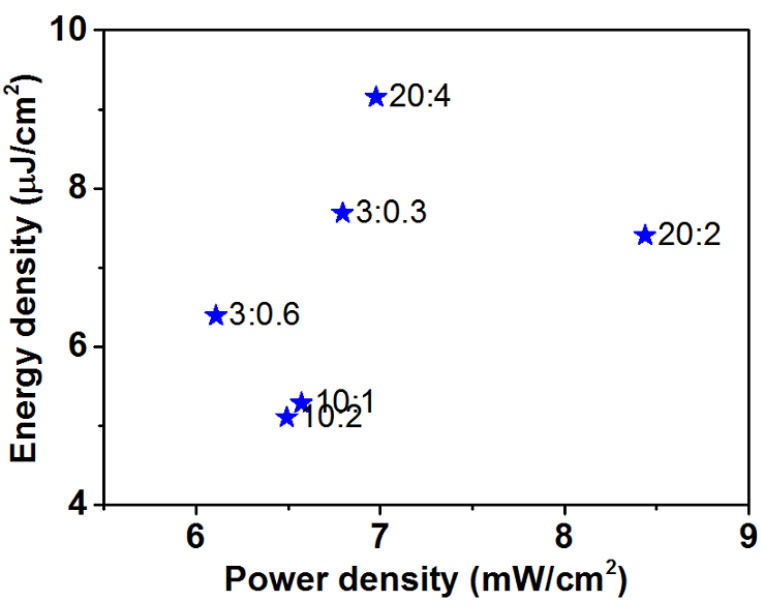

Fig. 4. Energy density versus power density plot for the devices.

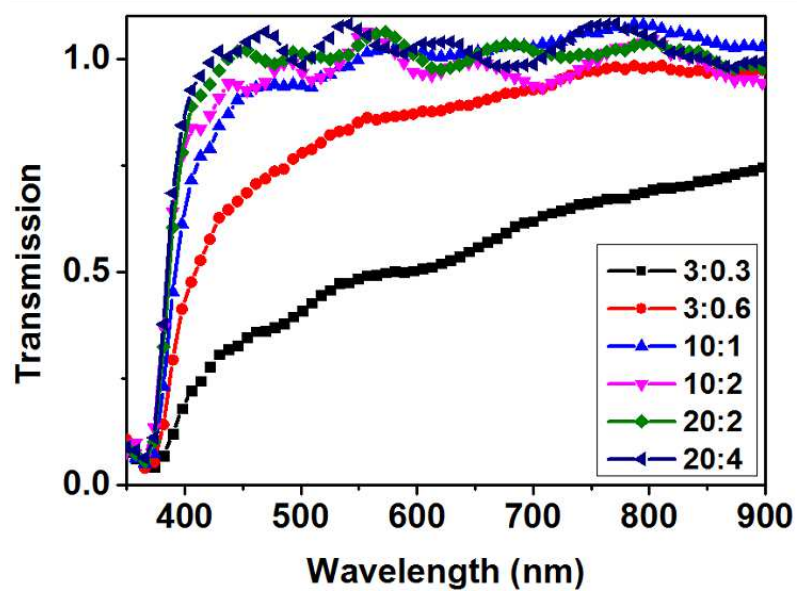

Fig. 5. Transmission versus light wavelength plots for the supercapacitors.

The power density of a supercapacitor can be described by the equation

$$
P=\frac{V_{0}^{2}}{4 E S R} \mathrm{~J} / \mathrm{cm}^{2},
$$

where $V_{0}$ is the bias range, equal to $1 \mathrm{~V}$ in this case and ESR is the series resistance. The energy density is given by

$$
E=\frac{C_{e f f} V_{0}^{2}}{2} \mathrm{~W} / \mathrm{cm}^{2} .
$$

The high power of the "20:2" sample is thus related to its lowest series resistance, as measured by EIS spectroscopy. The high energy density of the " $20: 4$ " sample relates to its highest effective capacitance.

The spectral optical transmission of the devices is presented in Fig. 5. It can be seen that the sample "3:0.3" has the lowest transmission characteristic, followed by the sample "3:0.6". The other samples present the value of the transmission coefficient close to 1 in the visible range. The fringes in the transmission relate to light interference 
in the device. The changes in the transmission coefficients can be related to the changes in the morphology. The lowest transmission is observed for the samples with the largest crystallites. On the other hand, the densification of the electrodes, as well as the formation of aligned nanocolumns leads to reduced light scattering and more transparency.

\section{Conclusions}

We showed that by controlling the morphology of ZnO-based nanostructured electrodes, the performance of transparent supercapacitors fabricated using these electrodes can be tuned. In order to achieve high effective capacitances, the morphology of the electrodes should either be a highly hierarchical one with large pores branching out into ever finer ones, or an aligned, nanocolumn morphology is beneficial. For the studied $\mathrm{ZnO}$ electrodes, the highest effective capacitances of $18.3 \mu \mathrm{F} / \mathrm{cm}^{2}$ were obtained for the nanowire-like morphologies, while the lowest for a dense morphologies with only small pores $-10.2 \mu \mathrm{F} / \mathrm{cm}^{2}$. Taking into account also the transparency, higher for the nanowire-like morphologies, it has to be concluded that the optimal $\mathrm{ZnO}$ morphology for transparent supercapacitor applications is the nanowire one.

\section{Acknowledgments}

This majority of this research was supported by the National Centre for Research and Development in the frames of the Lider V Programme through the project "Nanocoral zinc oxide-based supercapacitors for transparent electronics (NACZO)", contract: LIDER/030/615/L-5/ NCBR/2014 [11].

\section{References}

[1] K. Gao, Z. Shao, X. Wu, X. Wang, Y. Zhang, W. Wang, F. Wang, Nanoscale 5, 5307 (2013).

[2] J. Ge, G. Cheng, L. Chen, Nanoscale 3, 3084 (2011).

[3] P. Kanninen, N. Dang Luong, L.H. Sinh, I.V. Anoshkin, A. Tsapenko, J. Seppälä, A.G. Nasibulin, T. Kallio, Nanotechnology 27, 235403 (2016).

[4] X.Y. Liu, Y.Q. Gao, G.W. Yang, Nanoscale 8, 4227 (2016).

[5] G. Zhang, H. Wu, X. Wang, T. Wang, C. Liu, Nanotechnology 27, 105204 (2016).

[6] M.A. Borysiewicz, E. Dynowska, V. Kolkovsky, J. Dyczewski, M. Wielgus, E. Kamińska, A. Piotrowska, Phys. Status Solidi A 209, 2463 (2012).

[7] M. Masłyk, M.A. Borysiewicz, M. Wzorek, T. Wojciechowski, M. Kwoka, E. Kamińska, Appl. Surf. Sci. 389, 287 (2016).

[8] A. Janotti, C.G. van de Walle, Rep. Prog. Phys. 72, 126501 (2009).

[9] G. Wang, X. Lu, Y. Ling, T. Zhai, H. Wang, Y. Tong, Y. Li, ACS Nano 6, 10296 (2012).

[10] F. Beguin, E. Frąckowiak, Supercapacitors: Materials, Systems and Applications, Wiley-VCH Verlag, Weinheim 2013.

[11] NACZO, Nanocoral zinc oxide-based supercapacitors for transparent electronics, accessed at 12.27.2016. 